\title{
Neutron Shielding Simulations and Muon-induced Neutrons
}

\author{
Laura Vanhoefer*, \\ Max-Planck-Institut für Physik, Föhringer Ring 6, D-80805 München, Germany and \\ Excellence Cluster Universe, Boltzmannstr. 2, D-85748, Garching, Germany \\ E-mail: Lvanhoefdmpp.mpg.de
}

\section{Iris Abt, Allen Caldwell, Christopher Gooch, Béla Majorovits, Matteo Palermo, Oliver Schulz \\ Max-Planck-Institut für Physik, Föhringer Ring 6, D-80805 München, Germany}

\begin{abstract}
Neutrons can create background in experiments built to search for rare events like neutrinoless double beta decay. They can generate long lived isotopes in the materials used in the experiment. Although neutrons can be shielded during storage and transportation, muons penetrate and can produce neutrons inside the shield and the material to be shielded. Since the expected signal rates are extremely low, understanding and reducing of background plays a crucial role for rare event searches. Therefore it is important to know the shielding properties and the muon-induced neutron rate of the shielding materials.

To determine the shielding properties of different materials, cosmic-ray neutrons and muons were simulated with the GEANT4 based framework MaGe. Since the angular distribution at sea level is not precisely known, different neutron angular distributions were evaluated. In addition, the influence of neutron back-scattering was investigated.
\end{abstract}

XVI International Workshop on Neutrino Telescopes,

2-6 March 2015

Palazzo Franchetti - Istituto Veneto, Venice, Italy

\footnotetext{
* Speaker.
} 


\section{Introduction}

Neutrons as well as muons are created in the atmosphere by cosmic rays. They can create background in experiments built to search for rare events like neutrinoless double beta decays. Since the expected signal rates are extremely low, the understanding and reducing of background plays a crucial role. Neutrons contribute to the background due to production of long-lived isotopes in materials used in the experiment. Muons can also create neutrons when they penetrate the material. Therefore it is important to know the shielding properties and the muon-induced neutron rate for background estimation.

Cosmic-ray neutrons and muons penetrating through different material blocks were simulated using the GEANT4 based framework MaGe. In section \, the cosmic-ray neutron simulations are discussed. The effect of backscattering and different neutron angular distributions are quantified in section [3. The muon-induced neutron simulations are discussed in section $[$.

\section{Cosmic-ray Neutron Simulations}

For the cosmic-ray neutron shielding simulations, the measured neutron energy spectrum of M.S. Gordon et al. [四] was used. Downwards moving neutrons penetrating blocks with different thicknesses between $1 \mathrm{~m}$ and $20 \mathrm{~m}$ in $1 \mathrm{~m}$ steps were simulated for plastic, water, soil and steel. Since steel is denser than the other materials, additional thicknesses in the $\mathrm{cm}$ and $\mathrm{dm}$ range were also simulated for steel. The total number of outgoing neutrons from each slab normalized to the number of arriving neutrons as a function of shielding depth in terms of mwe is shown in Fig. $\square$. It can be clearly seen that for steel more neutrons are produced for small block thicknesses. Only after a certain depth (depending on the material) there are less neutrons than neutrons which have arrived.

Since the average atomic number A and the densities of plastic and water are similar, their shielding properties are similar as well, which can be seen in Fig. W. It is apparent that the total neutron flux is only reduced with respect to the incoming neutron flux, if the block is thick enough. A fit was applied to get the shielding index $s$ :

$$
y=10^{s x+a}
$$

with the depth $x$ in terms of $1 / \mathrm{m}$, the logarithmic neutron flux $y(x)$ and a fit constant $a$. The results

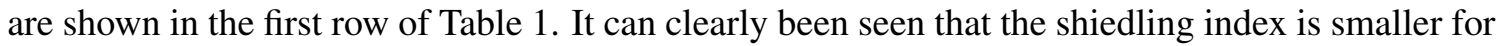
materials with higher average A. Different angular distributions are discussed in the next section.

\begin{tabular}{|c|c|c|c|c|}
\hline Angular distribution & Plastic & Water & Soil & Steel \\
\hline Only downwards going neutrons & $-0.354 \pm 0.003$ & $-0.372 \pm 0.003$ & $-0.57 \pm 0.01$ & $-1.90 \pm 0.03$ \\
\hline High energtic neutrons & $-0.366 \pm 0.002$ & $-0.397 \pm 0.007$ & $-0.584 \pm 0.007$ & $-2.01 \pm 0.03$ \\
\hline Isotropic & $-0.388 \pm 0.005$ & $-0.418 \pm 0.005$ & $-0.611 \pm 0.009$ & $-2.22 \pm 0.03$ \\
\hline
\end{tabular}

Table 1: Shielding index $s[1 / \mathrm{m}]$ for different materials and angular distributions.

\section{Backscattering and Angular Distribution Effects for Neutrons}

Three different angular distibutions were simulated to estimate the influence of the angular distibution: vertically downwards going neutrons, an isotropic distribution and a distribution sug- 

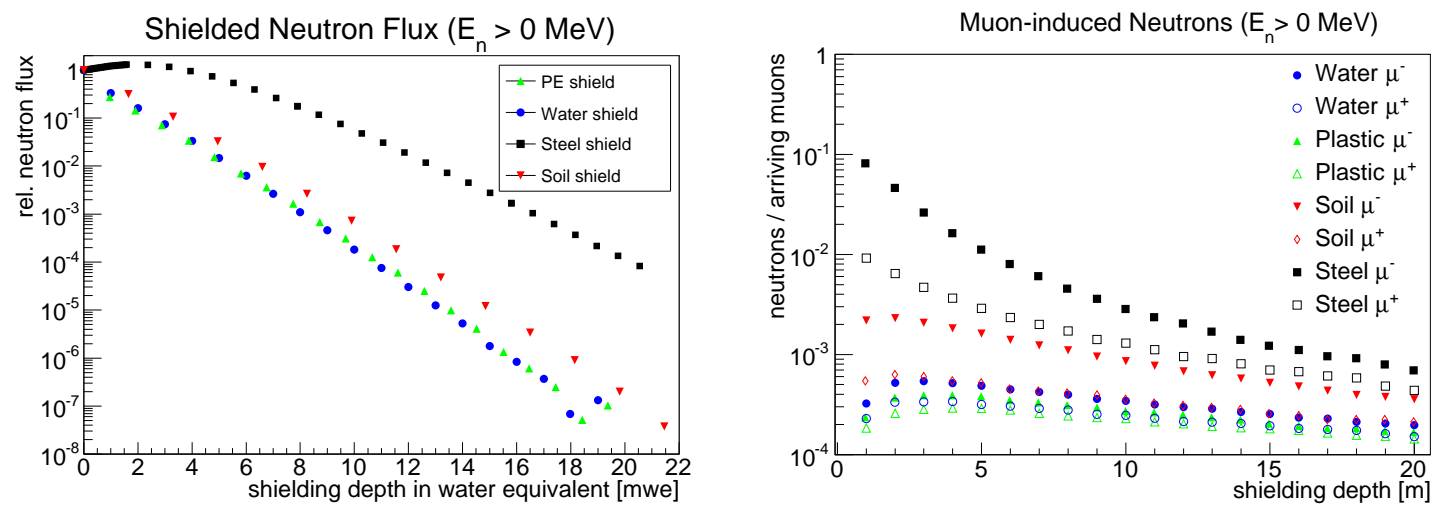

Figure 1: Neutron flux at the end of different material Figure 2: Muon-induced neutron flux at the end of blocks with different thicknesses. different material blocks with different thicknesses.

gested by [[] for high energetic neutrons $(\mathrm{E} \geq 10 \mathrm{MeV})$ :

$$
J(\theta)=J_{0} \exp [\alpha(1-\cos \theta)]
$$

with $\alpha=-2.5$, the flux in the vertical direction $J_{0}$ and the nadir angle $\theta$. The number of outgoing neutrons were counted for the different block thicknesses and the fit from Eq. 2 . Was applied. The results can be seen in Table $\mathbb{W}$. There are two extreme cases: only down going neutrons and an isotropic distribution. The shiedling index is the lowest for the isotropic distribution, since the neutrons need to penetrate on average a larger distance to leave the block and more neutrons are shielded. If the neutrons are only going downwards, they need to penetrate on average the smallest distance. Therefore the shielding index is larger for this distribution.

The influence of the chosen angular distribution is smaller than the influence of the chosen material. The shielding index is smallest for the material with highest average A for all three angular distributions.

Also the effect of backscattering was investigated. Since neutrons scatter inside the block, they can scatter back which leads to multiple counting of neutrons as they multiply cross horizontal the surface. To quantify the effect of backscattering, the outgoing neutrons at the end from blocks with different thicknesses were compared with the number of neutrons at the corresponding depth of a $20 \mathrm{~m}$ block. The result is shown in Table $\square$. In one case, only downwards going neutrons were counted, in the other case neutrons crossing the surface in any direction were counted. Without backscattering, the ratio would be one. The larger the ratio the more neutrons are scattered back. The ratio is obviously larger if neutrons crossing the surface in both directions are counted. It can clearly seen from Table $\square$ that the backscattering effect is larger for materials with higher average A and that the effect is significant.

\begin{tabular}{|c|c|c|c|c|}
\hline Material & Plastic & Water & Soil & Steel \\
\hline All neutrons & $1.3552 \pm 0.0006$ & $1.4918 \pm 0.0005$ & $2.1718 \pm 0.0005$ & $5.340 \pm 0.002$ \\
\hline Down going neutrons & $1.1370 \pm 0.0005$ & $1.1899 \pm 0.0004$ & $1.4723 \pm 0.0004$ & $2.9322 \pm 0.0009$ \\
\hline
\end{tabular}

Table 2: Average ratio of the neutron number crossing a surface at depths from $1 \mathrm{~m}$ to $20 \mathrm{~m}$ in steps of $1 \mathrm{~m}$ inside a solid divided by the number of neutrons exiting a block with the corresponding thickness. 


\section{Muon-induced Neutron Simulations}

If muons penetrate materials, they can induce neutrons. To estimate the muon-induced neutron rate, the muon energy spectrum of B.C. Rastin [3] was used. Unlike the neutron angular distribution, the muon angular distribution is well known and can be described by $\cos ^{2}(\theta)$.

Muons $\left(\mu^{+}\right.$and $\left.\mu^{-}\right)$penetrating through blocks with different thicknesses for plastic, water, soil and steel were simulated. The neutrons leaving the block at the other end were counted and normalized to the number of arriving muons. The result is shown in Fig. $\downarrow$. It can be clearly seen that the amount of neutrons is different for $\mu^{+}$and $\mu^{-}$. More neutrons are produced by $\mu^{-}$.

The shielding of $\mu^{+}$and $\mu^{-}$is similar, since they undergo similar reactions. But only $\mu^{-}$can be captured (in competition to muon decay) producing neutrons. From [䧃] it can be seen, that the stopping muon rate gets lower for larger shielding depths. Therefore the difference between the induced neutrons is getting lower with larger depths, since the low energetic muons have been shielded.

Comparing Fig. $\square$ with Fig. [, it can be seen, that the muon-induced neutrons begin to dominate the overall flux at a certain depth.

\section{Conclusion}

The shielding properties for cosmic-ray neutrons were determined for different angular distributions for different material. The lower the average A of the material the better the shielding is per mwe. The effect of the different angular distributions investigated was found to be smaller than

the effect of different materials simulated. Also the effect of backscattering was investigated and found to be significant (especially for steel).

The number of muon-induced neutrons is larger for $\mu^{-}$than for $\mu^{+}$. The larger the depth the smaller the difference is. This can be explained by the stopping-muon rate which is getting smaller for larger depths.

\section{Acknowledgments}

This research was supported by the DFG cluster of excellence "Origin and Structure of the Universe" (www.universe-cluster.de).

\section{References}

[1] M. S. Gordon et al., Measurement of the Flux and Energy Spectrum of Cosmic-Ray Induced Neutrons on the Ground, IEEE Transactions on Nuclear Science 51 (2004).

[2] A. Nesterenok, Numerical calculations of cosmic ray cascade in the Earth's atmosphere - Results for nucleon spectra, Nuclear Instruments and Methods in Physics Research B 295 (2013)

[3] B. C. Rastin, An accurate measurement of the sea-level muon spectrum within the range 4 to $3000 \mathrm{GeV} / \mathrm{c}$, J. Phys. G: Nucl. Phys. 10 (1984).

[4] G.L. Cassiday, J. W. Keuffel and J. A. Thomson Calculation of the Stopping-Muon Rate Underground, Physical Review D 7 (1973). 